\title{
Long non-coding RNA LINK-A promotes glioma cell growth and invasion via lactate dehydrogenase $A$
}

\author{
DEJUN WU ${ }^{1}$, BING ZHAO ${ }^{1}$, XIAOYU CAO ${ }^{3}$ and JINGHAI WAN ${ }^{1,2}$ \\ ${ }^{1}$ Department of Neurosurgery, The Second Affiliated Hospital of Anhui Medical University, Hefei, Anhui 230601; \\ ${ }^{2}$ Department of Neurosurgery, Cancer Institute and Hospital, Chinese Academy of Medical Sciences, Beijing 100021; \\ ${ }^{3}$ Department of Neurosurgery, Beijing Tiantan Hospital, Capital Medical University, Beijing 100050, P.R. China
}

Received November 10, 2016; Accepted June 6, 2017

DOI: $10.3892 /$ or.2017.5806

\begin{abstract}
Long non-coding RNAs (lncRNAs) have recently been identified in mammals as new modulators of cancer origin and progression participating in various cellular processes. Long intergenic non-coding RNA for kinase activation (LINKA), belonging to one of the intergenic lncRNAs, was reported to regulate signaling pathways correlated with triple-negative breast cancer. However, the expression and the functional role of LINK-A in glioma are still unclear. In the present study, we demonstrated that LINK-A was upregulated in human glioma cells compared with the expression noted in normal astrocytes. Knockdown of LINK-A inhibited cell proliferation, migration and invasion in U87 and U251 glioma cells. In addition, we found that lactate dehydrogenase A (LDH-A) was regulated by LINK-A, and enforced expression of LDH-A promoted glycolysis and proliferation in glioma cells. More importantly, we found that LDH-A was involved in the LINK-A-mediated proliferation and invasion of glioma cells. Collectively, these results provide new evidence of an important role for LINK-A in the development of glioma.
\end{abstract}

\section{Introduction}

Gliomas account for 30 to $40 \%$ of all intracranial tumors. Approximately half of all gliomas in adults are glioblastoma (GBM) the most aggressive subtype with a 5-year survival rate of less than $5 \%(1,2)$. Currently, some advances have

Correspondence to: Dr Jinghai Wan, Department of Neurosurgery, The Second Affiliated Hospital of Anhui Medical University, 678 Furong Road, Hefei, Anhui 230601, P.R. China

E-mail:wanjinghai@sina.com

Abbreviations: GBM, glioblastoma; lncRNAs, long non-coding RNAs; LINK-A, long intergenic non-coding RNA for kinase activation; HA, normal human astrocyte; RNAi, RNA interference; ncRNAs, non-coding RNAs; LDH-A, lactate dehydrogenase A

Key words: glioma, lncRNAs, LINK-A, LDH-A been achieved in regards to multimodal treatments, including surgical extirpation, local irradiation and conventional chemotherapy. However, the overall survival of most glioma patients remains poor, particularly for GBM patients $(3,4)$. Mounting efforts have been made to explore the molecules and signaling pathways involved in glioma cell proliferation, migration and invasion $(5,6)$. However, the mechanisms are still poorly understood, and the identification of key molecules that show a potential effect on glioma development is still imperative.

Long non-coding RNAs (IncRNAs) are defined as endogenous cellular RNAs more than 200 nucleotides long, which lack a functional open reading frame (7). Accumulating evidence suggests that 1 ncRNAs are pivotal regulatory molecules that are implicated in diverse biological processes, including epigenetic, transcriptional and post-transcriptional regulatory mechanisms (8-10). It has been found that numerous lncRNAs play central roles in the tumor-related gene regulatory system, and dysregulation of their expression is thought to contribute to tumor cell proliferation, invasion and metastasis (11-13). Several lines of evidence point to the etiologic role of dysregulated lncRNAs in glioma, including CRNDE, CASC2, HOTAIR, GAS5 and MEG3 (14-18).

Recently, Lin et al reported that long intergenic noncoding RNA for kinase activation (LINK-A) is critical to the growth factor-induced normoxic HIFl $\alpha$ signaling pathway in triple-negative breast cancer (19). However, far less is known concerning the role of LINK-A in glioma as well as the underlying mechanisms. To expand our knowledge regarding the biological function of LINK-A in glioma cells, the present study was designed in an attempt to identify the contribution of LINK-A to the proliferation and invasion of glioma cells, thereby providing novel therapeutic strategies for gliomas.

\section{Materials and methods}

Cell culture procedures. U87 and U251 glioma cells, and normal human astrocytes (HAs) were purchased from the American Type Culture Collection (ATCC; Manassas, VA, USA). Cells were cultured in Dulbecco's modified Eagle's medium (DMEM), supplemented with $10 \%$ heat-inactivated fetal bovine serum (FBS) and $100 \mathrm{U} / \mathrm{ml}$ penicillin/streptomycin. Cell cultures were maintained at $37^{\circ} \mathrm{C}$ in a humidified atmosphere of $5 \% \mathrm{CO}_{2}$. 
RNA interference (RNAi) analysis and plasmid construction. The following short hairpin RNA (shRNA) was used to target human LINK-A: 5'-TTACTGAGGTTGAATATGT-3'. Recombinant lentiviruses expressing sh-LINK-A or sh-control were produced. The lactate dehydrogenase A (LDH-A) sequences were synthesized and subcloned into the pCDNA3.1 vector. The pCDNA constructs or the empty vector were transfected into glioma cells cultured on 6-well plates according to the manufacturer's instructions.

Real-time PCR analysis. Total RNA was extracted from glioma cells using TRIzol reagent (Invitrogen, Carlsbad, CA, USA). The first-strand cDNA was synthesized from total RNA using the ThermoScript RT-PCR system. In brief, each PCR reaction mixture containing $10 \mu \mathrm{l}$ of $2 \mathrm{X}$ SYBR-Green Master Mix, $1 \mu \mathrm{l}$ of sense and antisense primers $(5 \mu \mathrm{mol} / \mu \mathrm{l})$ and $1 \mu \mathrm{l}$ of cDNA (10 ng), was run for 45 cycles with denaturation at $95^{\circ} \mathrm{C}$ for $15 \mathrm{sec}$, annealing at $60^{\circ} \mathrm{C}$ for $30 \mathrm{sec}$ and extension at $72^{\circ} \mathrm{C}$ for $30 \mathrm{sec}$ in a total volume of $20 \mu \mathrm{l}$. For relative quantification, $2^{-\Delta \Delta \mathrm{Ct}}$ was calculated and used as an indication of the relative expression levels, which were calculated by subtracting CT values of the control gene from the CT values of LINK-A and LDH-A. The primer sequences for PCR amplification were: LINK-A, 5'-TTCCCCCATTTTTCCTTTTC-3' and 5'-CTCTGGTTGGGTGACTGGTT-3'; LDH-A, 5'-TGTGCC TGTATGGAGTGGAA-3' and 5'-AGCACTCTCAACCACC TGCT-3'. GAPDH was applied as an internal control. The primer sequences of GAPDH were: 5'-AGCAAGAGCACAAG AGGAAG-3' and 5'-GGTTGAGCACAGGGTACTTT-3'.

MTT assay. U87 and U251 glioma cells were trypsinized, resuspended, seeded into a 96-well plate at a concentration of 2,000 cells $/$ well, and incubated at $37^{\circ} \mathrm{C}$. The number of viable cells was measured at daily intervals. At each time point, $10 \mu \mathrm{l}$ of $5 \mathrm{mg} / \mathrm{ml}$ MTT (DingGuo Biotechnology, Co., Ltd., Beijing, China) was added, and incubation was continued for $4 \mathrm{~h}$. Then, the medium was removed carefully and $100 \mu \mathrm{l}$ dimethyl sulfoxide was added at the end of the incubation. The absorbance was measured at $490 \mathrm{~nm}$ on a spectrophotometer.

Colony formation assay. U87 and U251 glioma cells were seeded into 6-well plates. The medium was replaced at regular time intervals. After 14 days of culture at $37^{\circ} \mathrm{C}$, the natural colonies were washed with phosphate-buffered saline (PBS) and fixed with $4 \%$ paraformaldehyde for $30 \mathrm{~min}$ at room temperature. The colonies were then stained with methylene blue for $10 \mathrm{~min}$, washed with water and air-dried. The total number of colonies with $>50$ cells was counted under fluorescence microscopy.

Scratch wound assay. U87 and U251 glioma cells were infected with sh-LINK-A or sh-control. Wounds were created in adherent cells using a $20 \mu \mathrm{l}$ pipette tip, $48 \mathrm{~h}$ after infection. The cells were then washed 3 times with PBS to remove any free-floating cells and debris. Medium without serum was added, and the cells were incubated under normal conditions. Wound healing was observed after $24 \mathrm{~h}$ under light microscopy. Representative scrape lines were photographed using digital microscopy after the culture inserts were removed. Each experiment was repeated in triplicate.
Invasion assays. Cells $\left(5 \times 10^{5}\right)$ were seeded on the top side of a polycarbonate Transwell filter coated with Matrigel (for Transwell matrix penetration assay) in the upper chamber of the QCM $^{\mathrm{TM}}$ 24-Well Cell Invasion Assay (Cell Biolabs, Inc., San Diego, CA, USA). For the invasion assay, cells were suspended in medium without serum, and medium supplemented with serum was used as a chemoattractant in the lower chamber. The cells were incubated at $37^{\circ} \mathrm{C}$ for $24 \mathrm{~h}$. The non-invasive cells in the top chambers were removed with cotton swabs. The invaded cells on the lower membrane surface were fixed with methanol and stained with crystal violet. Cells were counted visually in 5 random fields under a light microscope (10X objective lens). In addition, invaded cells were dissociated, lysed and quantified at $570 \mathrm{~nm}$ using a spectrophotometer.

Western blotting. U87 and U251 glioma cells were lysed with RIPA lysis buffer (Beyotime, Beijing, China). Whole extracts were prepared, and protein concentrations were determined using the BCA protein assay kit (Boster, Wuhan, China). Whole-cell extracts $(20 \mu \mathrm{g})$ were then fractionated by electrophoresis through $12 \%$ sodium dodecyl sulfate-polyacrylamide gel electrophoresis (SDS-PAGE). Gels were run at $120 \mathrm{~V}$ for $2 \mathrm{~h}$ before transfer onto a polyvinylidene difluoride (PVDF) membrane (Millipore Corporation Billerica, MA, USA). After blocking against non-specific protein binding, nitrocellulose blots were incubated for $1 \mathrm{~h}$ with primary antibodies diluted in TBS/Tween-20 (0.075\% Tween-20) containing 3\% MARVEL. An-LDH-A (Santa Cruz Biotechnology, Santa Cruz, CA, USA) was diluted at 1:500. Following incubation with the primary antibody, blots were washed 3 times in TBS/Tween-20 before incubation for $1 \mathrm{~h}$ with goat anti-mouse horseradish peroxidase-conjugated antibody at a 1:10,000 dilution in TBS/Tween-20 containing 5\% milk. After extensive washing in TBS/Tween-20, the blots were rinsed with distilled water and proteins were detected using the enhanced chemiluminescence system. Proteins were visualized with a chemiluminescent (ECL) kit (ECL Plus; Thermo Fisher Scientific, Inc., Waltham, MA, USA).

Measurement of glucose consumption and lactate production. LDHA-expressing vector or the emplty vector was transfected into U87 and U251 glioma cells. Cell culture media were collected $48 \mathrm{~h}$ after the transfection. Lactate production and glucose uptake were measured using a lactate assay kit (Sigma, St. Louis, MO, USA) and Amplex Red Glucose/ Glucose Oxidase Assay kit (Invitrogen), respectively. The results were normalized according to total cellular protein amounts.

Statistical analysis. All data are expressed as mean \pm SD of 3 independent experiments, in which each assay was performed in triplicate. Data were analyzed with SPSS 16.0 software. Evaluation of the data was performed by Student's t-test (two-sided) and one-way ANOVA. $\mathrm{P}<0.05$ was considered statistically significant.

\section{Results}

LINK-A is upregulated in glioma cells. The expression levels of LINK-A in glioma cell lines (U87 and U251) and normal 
A

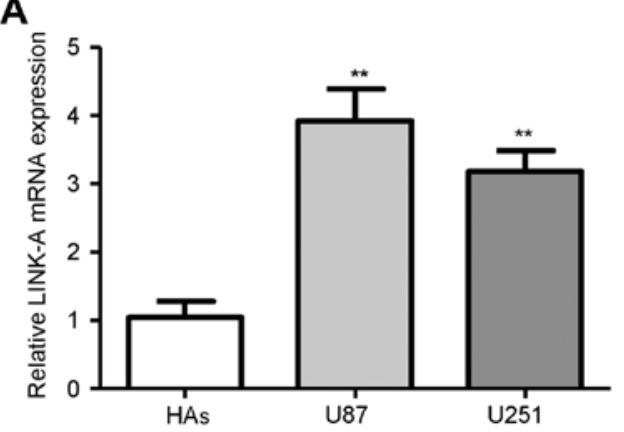

B

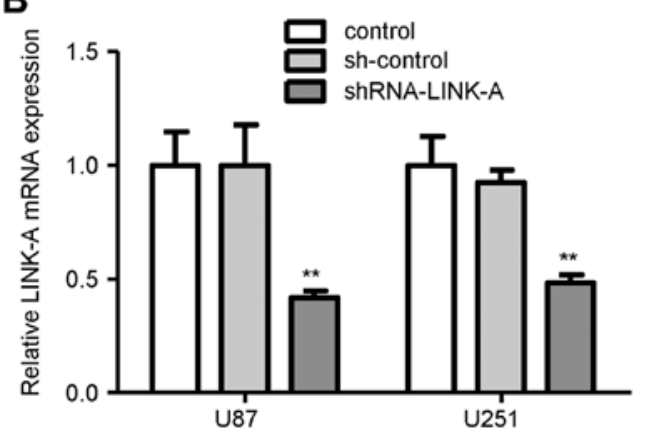

Figure 1. LINK-A is highly expressed in human glioma cells. (A) RT-qPCR analysis of LINK-A expression in glioma cell lines (U87 and U251) and normal human astrocytes (HAs); ${ }^{* *} \mathrm{P}<0.01$ vs. the HA group. (B) Relative expression levels of LINK-A after glioma cells were infected with shRNA-LINK-A and sh-control; ${ }^{* *} \mathrm{P}<0.01$ vs. the sh-control group. Data are presented as mean \pm SD from 3 independent experiments.

A

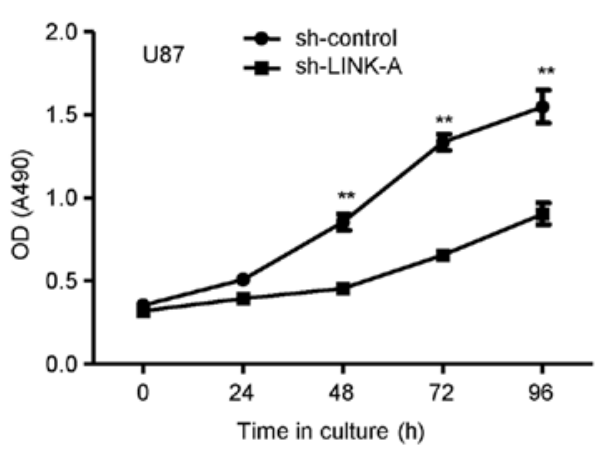

C

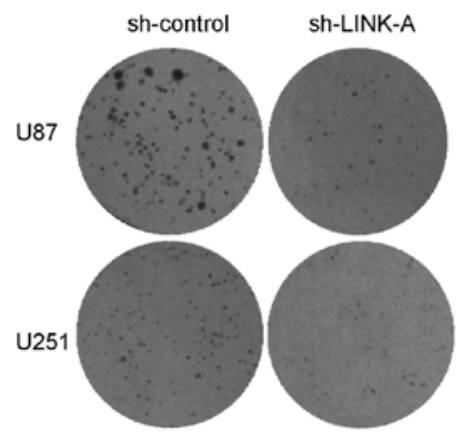

B
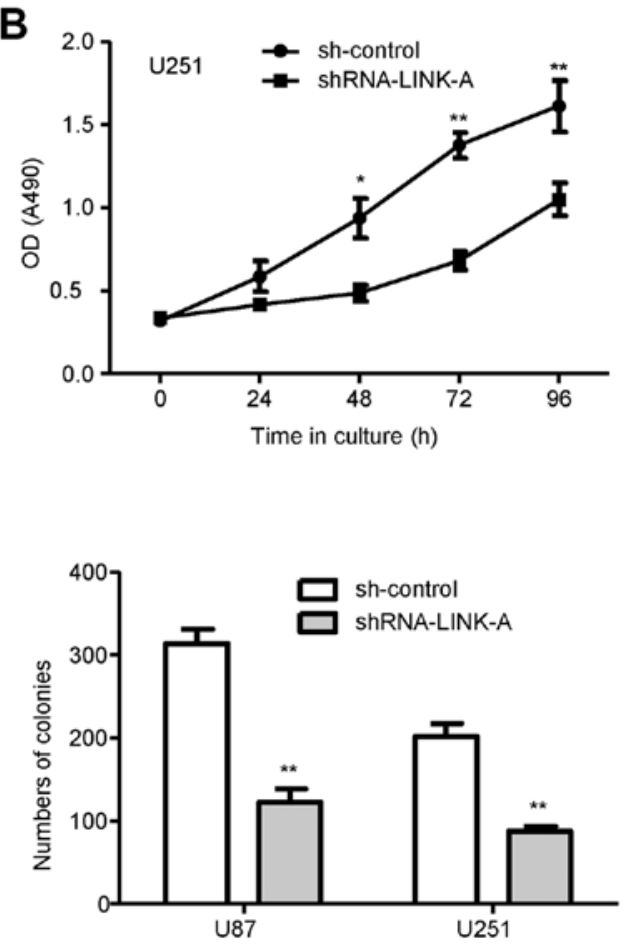

Figure 2. Knockdown of LINK-A inhibits the proliferation and colony formation of glioma cells in vitro. (A and B) MTT assay was performed to determine the proliferative effect of shRNA-LINK-A- and sh-control-infected U87 and U251 cells; ${ }^{*} \mathrm{P}<0.05,{ }^{* *} \mathrm{P}<0.01$ vs. the sh-control group. (C) Colony formation assay was performed to detect the proliferation of U87 and U251 cells after infection with shRNA-LINK-A and sh-control; ${ }^{* *} \mathrm{P}<0.01$ vs. the sh-control group. Data are presented as mean \pm SD from 3 independent experiments.

HAs were examined. As shown in Fig. 1A, the mRNA expression of LINK-A was higher in the glioma cell lines than that in the HAs, suggesting that LINK-A upregulation may play important roles in human glioma.

LINK-A promotes cell growth in glioma cells. To further investigate the function of LINK-A in glioma cells, a lentivirus carrying a specific shRNA against LINK-A (shRNA-LINK-A) to knockdown its expression was infected into U87 and U251 glioma cells. U87 and U251 cells with non-target shRNA (sh-control) served as the control. As shown in Fig. 1B, knockdown of LINK-A significantly decreased LINK-A mRNA expression in the U87 glioma cells compared with that in the sh-control group. Similar results were found in the U251 glioma cells (Fig. 1B). Then, we performed MTT assays to detect the effects of LINK-A on glioma cell proliferation. As shown in Fig. 2A and B, U87 and U251 glioma cells transfected with shRNA-LINK-A exhibited a lower proliferative rate, in comparison with that noted in the respective sh-control groups. To examine whether LINK-A has an influence on the colony-forming capacity of glioma cells, a colony formation assay was performed in the U87 and U251 glioma cells. The numbers of colonies formed in the shRNA-LINK-A groups were significantly decreased when compared with the numbers in the sh-control groups (Fig. 2C), suggesting that the reduced expression of LINK-A significantly inhibited colony formation in the glioma cells.

LINK-A promotes glioma cell migration and invasion. To ascertain whether LINK-A is involved in the migration 
A
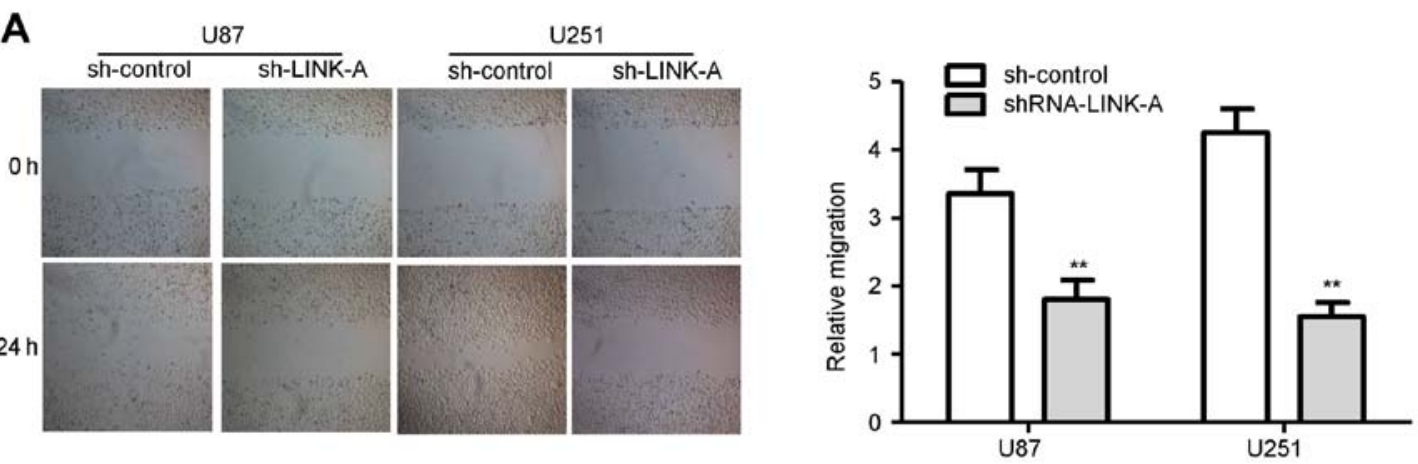

B
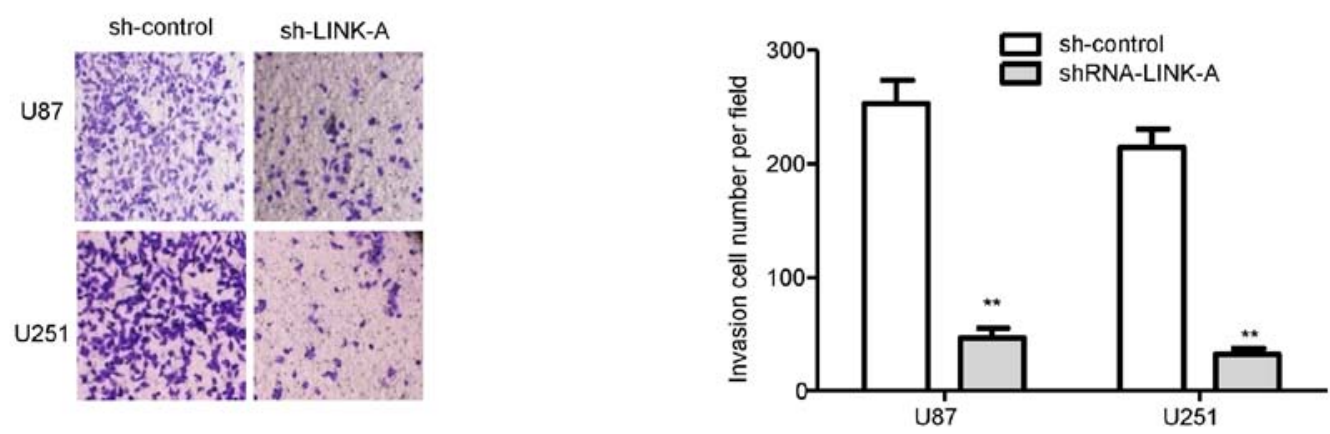

Figure 3. (A) Wound healing assay to evaluate the effect of LINK-A on cell migration in U87 and U251 cells; ${ }^{* *} \mathrm{P}<0.01<0.05$ vs. the sh-control group. (B) Invasion assay in U87 and U251 cells was performed to determined cell invasiveness after infection with shRNA-LINK-A and sh-control; ${ }^{* *} \mathrm{P}<0.01$ vs. the sh-control group. Data are presented as mean \pm SD from 3 independent experiments.
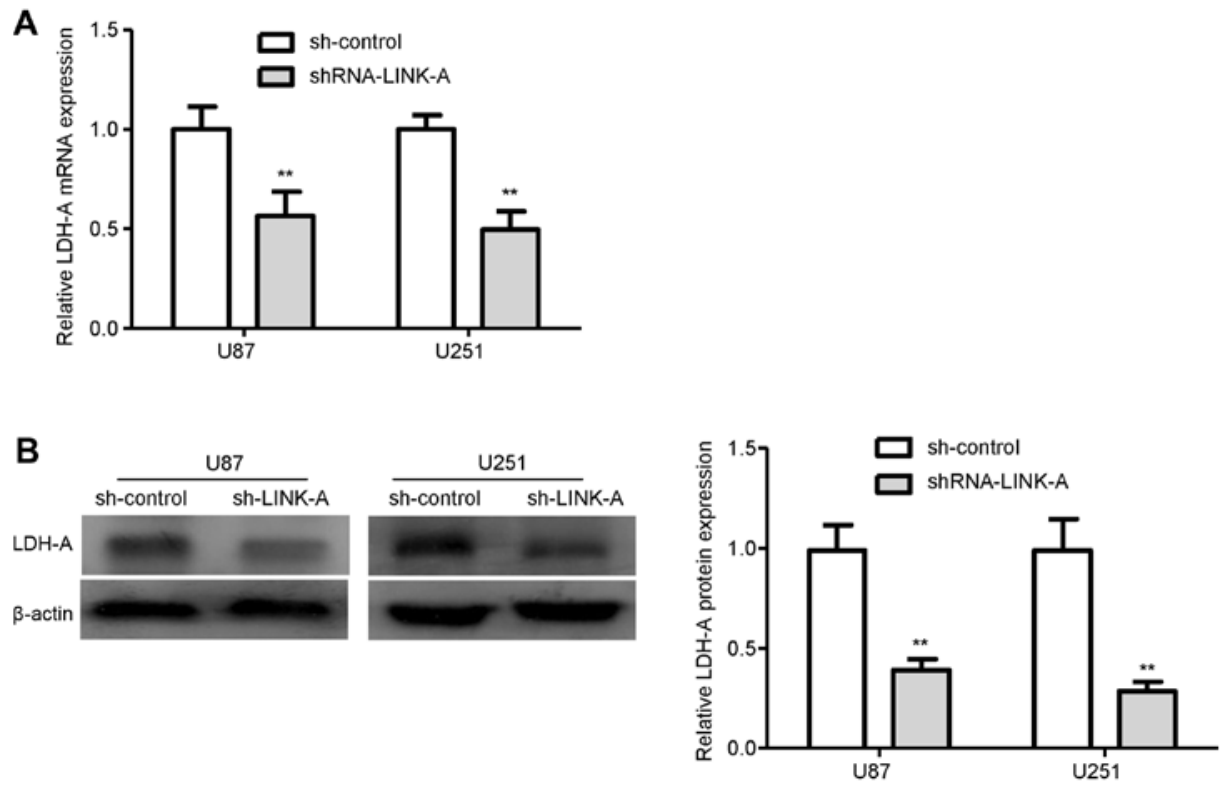

Figure 4. LINK-A knockdown reduces the LDH-A expression levels. (A) Relative mRNA expression of LDH-A in U87 and U251 cells following infection with shRNA-LINK-A and sh-control; * $\mathrm{P}<0.01$ vs. the sh-control group. (B) Relative protein expression of LDH-A in U87 and U251 cells infected with shRNA-LINK-A and sh-control; ${ }^{* *} \mathrm{P}<0.01$ vs. the sh-control group. Data are presented as mean $\pm \mathrm{SD}$ from 3 independent experiments.

and invasion of glioma cells, we performed wound healing and invasion assays. The migration assays showed that the migratory rate of the U87 and U251 glioma cells in the shRNALINK-A groups was significantly reduced in comparison with the sh-control groups (Fig. 3A). Similarly, Transwell invasion assays confirmed that LINK-A knockdown reduced the invasion ability of the U87 and U251 glioma cells (Fig. 3B).
Suppression of LDH-A by LINK-A knockdown. To explore the molecular mechanism by which LINK-A exerts biological function in glioma cells, we performed qRT-PCR analysis to investigate the effects of LINK-A knockdown on LDH-A, which is frequent aberrantly activated in cancer (20). qRT-PCR analysis showed that the levels of LDH-A mRNA expression were markedly reduced in the sh-LINK-A-infected U87 and 
A



C

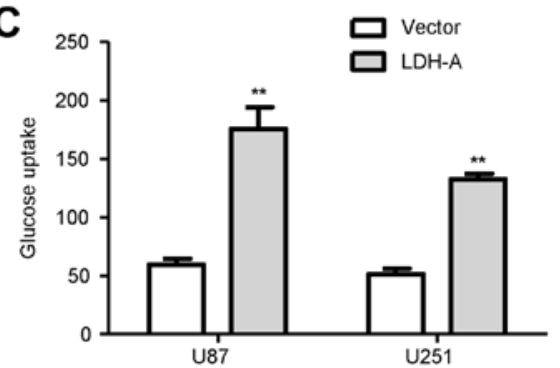

D

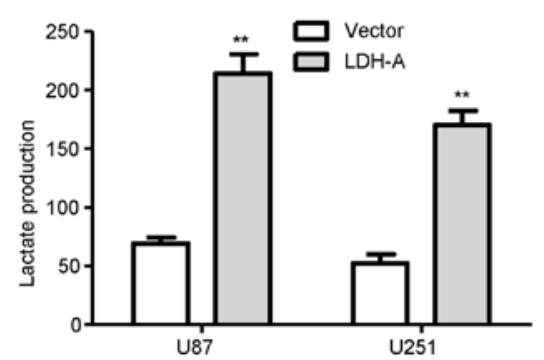

B

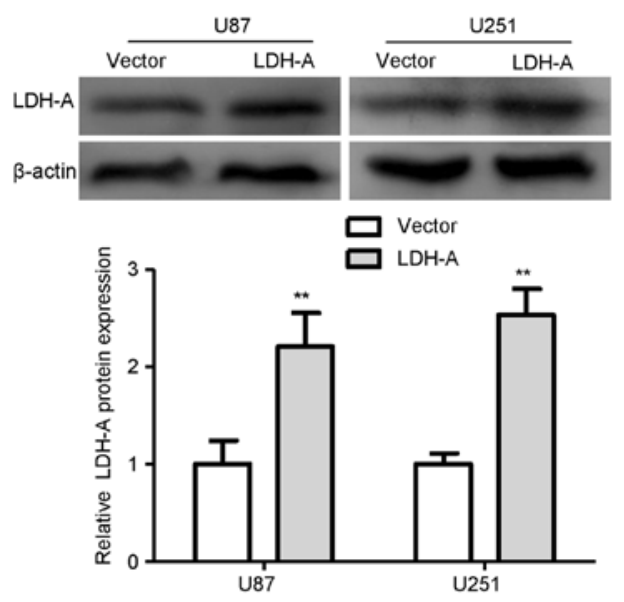

E

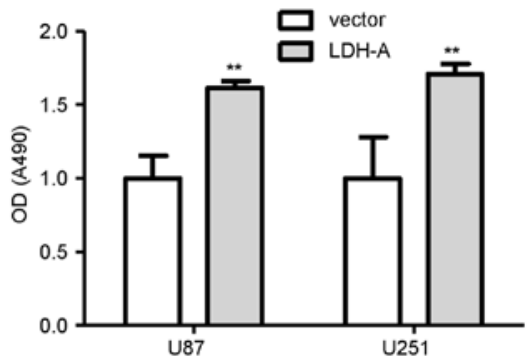

Figure 5. LDHA promotes glycolysis and cell proliferation in vitro and in vivo. (A) Relative mRNA expression of LDH-A in U87 and U251 cells transfected with the LDHA-expression vector or empty vector; ${ }^{* *} \mathrm{P}<0.01$ vs. the empty vector group. (B) Relative protein expression of LDH-A in U87 and U251 cells transfected with the LDHA-expression vector or empty vector; ${ }^{* *} \mathrm{P}<0.01$ vs. the empty vector group. (C and $\left.\mathrm{D}\right) \mathrm{Levels}$ of glucose uptake and lactate production were measured in U87 and U251 cells transfected with the LDHA-expression vector or empty vector; ${ }^{* *} \mathrm{P}<0.01$ vs. the empty vector group. (E) MTT assay was performed to determine the proliferative effect of U87 and U251 cells transfected with the LDH-A-expression vector or empty vector; ${ }^{* *} \mathrm{P}<0.01$ vs. the empty vector group. Data are presented as mean \pm SD from 3 independent experiments.

U251 glioma cells compared with levels in the sh-control groups (Fig. 4A). Similarly, knockdown of LINK-A significantly reduced the protein expression of LDH-A in both the U87 and U251 glioma cells (Fig. 4B). These results indicated that LDH-A may be involved in the LINK-A-induced proliferation and invasion of glioma cells.

LDH-A promotes glycolysis and cell proliferation. To assess the biological effects of LDH-A in glioma, LDHAexpressing vector or the empty vector was transfected into U87 and U251 glioma cells, respectively. qRT-PCR and western blot analysis demonstrated that the transfection was successful (Fig. 5A and B). Next, we examined the differences in metabolic parameters and we found that increased expression of LDH-A largely influenced aerobic glycolysis in the glioma cells, e.g., increased glucose uptake and lactate production (Fig. 5C and D). To confirm the role of LDH-A in glioma cells, we performed a proliferation assay in glioma cells. We found that overexpression of LDH-A in the U87 and U251 glioma cells significantly promoted cell proliferation compared with that in the empty vector groups (Fig. 5E).

LDH-A mediates the tumor-suppressive effects of sh-LINK-A in glioma cells. To clarify whether the tumor-suppressive effects of shRNA-LINK-A were mediated by LDH-A, we transfected the LDH-A plasmid into the U87 and U251 glioma cells infected by shRNA-LINK-A. In rescue studies, cell proliferation assay results showed that shRNA-LINK-A inhibited glioma cell proliferation and LDH-A promoted glioma cell proliferation. Co-transfection of shRNA-LINK-A and the LDH-A plasmid showed that LDH-A rescued the decrease in glioma cell proliferation by shRNA-LINK-A (Fig. 6A). Colony formation assay was used to further assess the proliferation ability. As shown in Fig. 6B, overexpression of LDH-A rescued U87 and U251 glioma cell cloning capability inhibited by shRNA-LINK-A. Moreover, overexpression of LDH-A reversed the invasive ability in the LINK-A knockdown glioma cells (Fig. 6C). Therefore, these results indicate that the tumor oncogene function of LINK-A is via LDH-A in glioma cells.

\section{Discussion}

Genome-wide surveys have revealed that $~ 90 \%$ of the genome is actively transcribed into non-coding RNAs (ncRNAs), while $<2 \%$ of the genome sequences encode proteins (21). Although ncRNAs were initially argued to be spurious transcriptional noise, recent evidence suggests that the transcriptional noise of the genome may play a major biological role in cellular development and human diseases $(22,23)$. 

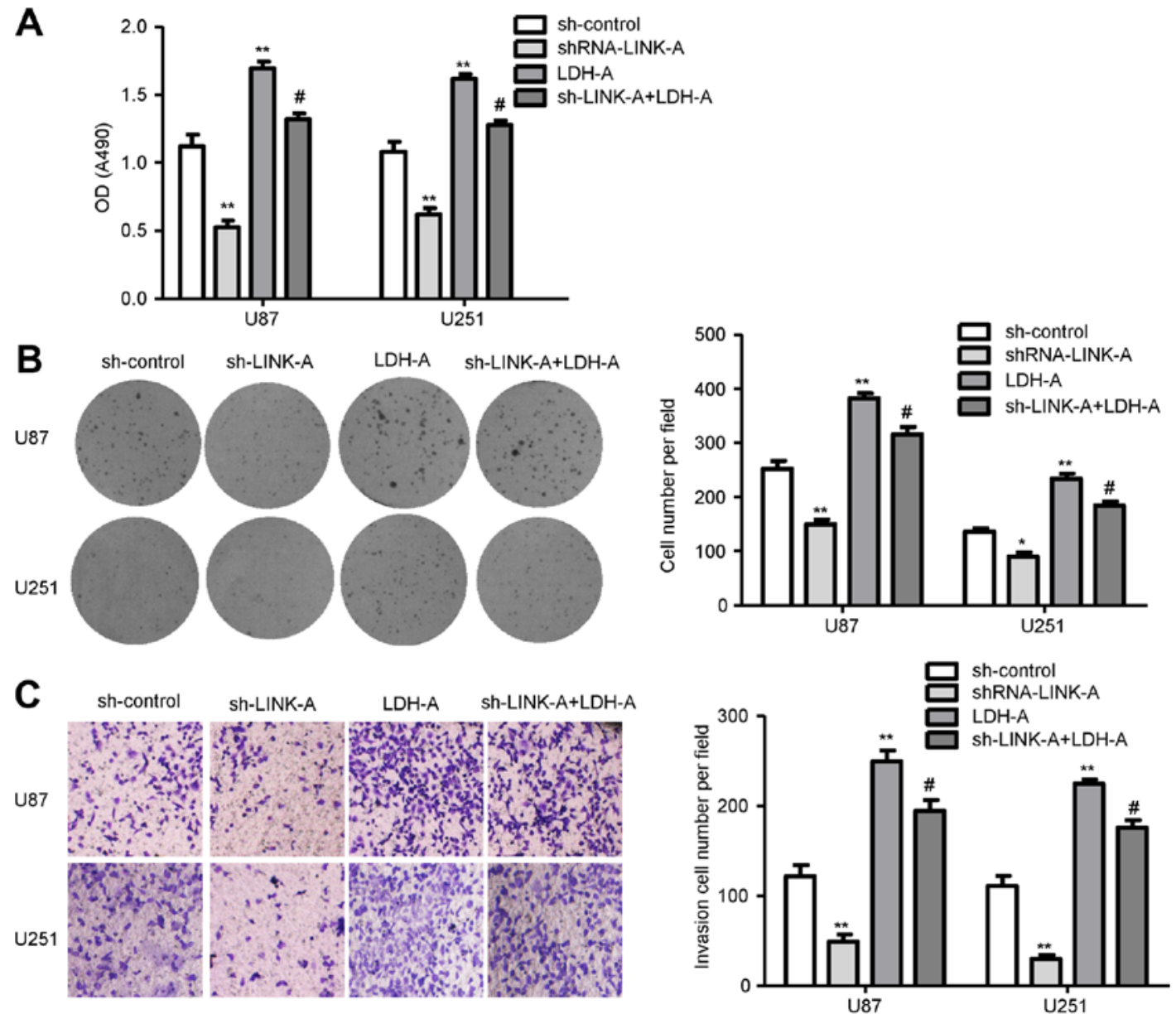

Figure 6. Overexpression of LDH-A abolishes the shRNA-LINK-A-induced inhibitory effects on glioma cells. (A) MTT assay was performed to determine the proliferation in the glioma cells after co-transfection with LDH-A and shRNA-LINK-A for $48 \mathrm{~h} ;{ }^{*} \mathrm{P}<0.05,{ }^{* *} \mathrm{P}<0.01$ vs. the empty vector group; ${ }^{\#} \mathrm{P}<0.05$ vs. the LDH-A group. (B) Colony formation assay was performed to detect the proliferation effect on U87 and U251 cells after co-transfection with LDH-A and shRNA-LINK-A (sh-LINK-A+LDH-A); ${ }^{*} \mathrm{P}<0.05,{ }^{* *} \mathrm{P}<0.01$ vs. the empty vector group; ${ }^{\mathrm{P}} \mathrm{P}<0.05$ vs. the LDH-A group. (C) Invasion assay in U87 and U251 cells was performed to determine cell invasiveness after co-transfection with sh-LINK-A+LDH-A; ${ }^{* *} \mathrm{P}<0.01$ vs. the empty vector group; $\mathrm{P}<0.05$ vs. the LDH-A group. Data are presented as mean \pm SD from 3 independent experiments.

The newly discovered lncRNAs, identified as one type of ncRNAs, are poorly conserved and capable to regulate gene expression at various levels $(24,25)$. Generally, IncRNAs have been involved in gene-regulatory roles, such as chromosome dosage-compensation, imprinting, epigenetic regulation, cell cycle control, nuclear and cytoplasmic trafficking, transcription, translation, splicing and cell differentiation $(23,26,27)$. Recently, it has been found that lncRNAs affect many cellular processes in tumor cells, such as cell cycle, proliferation, migration and invasion $(28,29)$.

During recent years, an accumulated number of studies have focused on the functional role of lncRNAs in tumorigenesis. Zhang et al reported that HOTAIR is a cell cycle-related lncRNA in human glioma, and its expression is closely related to glioma staging and poor prognosis (30). In addition, MALAT1 expression was lower than that in normal brain tissues, whereas overexpression of MALAT1 caused significant reduction in cell proliferation and invasion in vitro, and tumorigenicity in both subcutaneous and intracranial human glioma xenograft models. Furthermore, MALAT1-mediated tumor suppression in glioma cells may be via the attenuation of ERK/MAPK-mediated growth and MMP2-mediated invasiveness (31).
Long intergenic non-coding RNA for kinase activation (LINK-A), known as LOC339535, is a highly prognostic lncRNA in triple-negative breast cancer, which mediates HIF1 $\alpha$ phosphorylation, and then causes HIF1 $\alpha$ stabilization and activation of HIF1 $\alpha$ transcriptional programs (19). In the present study, we found that LINK-A was significantly upregulated in glioma cells. This result prompted us to speculate that downregulation of LINK-A may be essential for glioma cells, and its knockdown may suppress tumor growth of glioma. Via successful cell infection with shRNA-LINK-A, and the detection of glioma cell proliferation, migration and invasion, it was demonstrated that knockdown of LINK-A suppressed the growth of glioma cells. In addition, knockdown of LINK-A and the related assays were also performed to confirm the promoting effect of LINK-A on migration and invasion of glioma cells.

Lactate dehydrogenase A (LDH-A) is thought to be a major molecular mediator of the Warburg effect and to play a critical role in the metabolism of tumor cells (32-34). LDH-A increases the efficiency of the LDH complex, allowing the rapid flux via glycolysis that is responsible for the energy needs of rapidly proliferating cells (35). It has been reported that elevated levels of LDH-A are a hallmark of many tumors, including glioma, 
and is associated with the clinicopathological features and survival outcomes of patients (36-38). Inhibition of LDH-A typically results in accelerated oxygen consumption, reduced cell malignant transformation and markedly delayed tumor formation, indicating the underlying role of LDH-A in tumor initiation or maintenance $(20,39)$.

Recently, Lin et al reported that knockdown of LINK-A in triple-negative breast cancer cells markedly reduced the expression of LDH-A, and impaired glycolysis, suggesting that LDH-A may represent an important downstream effector of LINK-A (19). Therefore, we speculated that LDH-A was probably also regulated by LINK-A in glioma cells. Thus, we determined the levels of LDH-A in LINK-A-knockdown glioma cells. As a result, we found that LINK-A knockdown in glioma cells downregulated the LDH-A expression. Moreover, LDH-A facilitated glucose uptake, lactate production and markedly enhanced cell proliferation in glioma cells. These findings suggest that LDH-A may promote glioma malignant potential via the glycolysis pathway.

To investigate whether LDH-A is involved in the inhibition of cell proliferation and invasion regulated by LINK-A, shRNA-LINK-A was infected into glioma cells where LDH-A was overexpressed by transfection with pcDNA-LDH-A. LDH-A overexpression reversed the inhibitory effect mediated by LINK-A knockdown. Therefore, we conclude that LDH-A is involved in the proliferation and invasion of glioma cells influenced by LINK-A.

In conclusion, we found that knockdown of LINK-A inhibited glioma cell proliferation and invasion. Moreover, the involvement of LDH-A in the proliferation and invasion of glioma cells was mediated by LINK-A. Therefore, LINK-A may serve as an oncogenic lncRNA that promotes proliferation and invasion of glioma cells through LDH-A.

\section{Acknowledgements}

The present study was supported by the National Natural Science Foundation of China (grant no. 81470112).

\section{References}

1. Ostrom QT, Gittleman H, Farah P, Ondracek A, Chen Y, Wolinsky Y, Stroup NE, Kruchko C and Barnholtz-Sloan JS CBTRUS statistical report: Primary brain and central nervous system tumors diagnosed in the United States in 2006-2010. Neuro Oncol 15 (Suppl 2): ii1-ii56, 2013.

2. Wei J, Gabrusiewicz K and Heimberger A: The controversial role of microglia in malignant gliomas. Clin Dev Immunol 2013 285246, 2013.

3. Omuro A and DeAngelis LM: Glioblastoma and other malignant gliomas: A clinical review. JAMA 310: 1842-1850, 2013.

4. Van Meir EG, Hadjipanayis CG, Norden AD, Shu HK, Wen PY and Olson JJ: Exciting new advances in neuro-oncology: The avenue to a cure for malignant glioma. CA Cancer J Clin 60: 166-193, 2010

5. Gupta K and Salunke P: Molecular markers of glioma: An update on recent progress and perspectives. J Cancer Res Clin Oncol 138: 1971-1981, 2012.

6. Hochberg FH, Atai NA, Gonda D, Hughes MS, Mawejje B, Balaj L and Carter RS: Glioma diagnostics and biomarkers: An ongoing challenge in the field of medicine and science. Expert Rev Mol Diagn 14: 439-452, 2014

7. Chen LL and Carmichael GG: Long noncoding RNAs in mammalian cells: What, where, and why? Wiley Interdiscip Rev RNA 1: 2-21, 2010.
8. Jeon Y, Sarma K and Lee JT: New and Xisting regulatory mechanisms of $X$ chromosome inactivation. Curr Opin Genet Dev 22: 62-71, 2012.

9. Ørom UA, Derrien T, Beringer M, Gumireddy K, Gardini A, Bussotti G, Lai F, Zytnicki M, Notredame C, Huang Q, et al: Long noncoding RNAs with enhancer-like function in human cells. Cell 143: 46-58, 2010.

10. Quek XC, Thomson DW, Maag JL, Bartonicek N, Signal B, Clark MB, Gloss BS and Dinger ME: IncRNAdb v2 .0: Expanding the reference database for functional long noncoding RNAs. Nucleic Acids Res 43: D168-D173, 2015.

11. Gibb EA, Brown CJ and Lam WL: The functional role of long non-coding RNA in human carcinomas. Mol Cancer 10: 38 , 2011.

12. Spizzo R, Almeida MI, Colombatti A and Calin GA: Long non-coding RNAs and cancer: A new frontier of translational research? Oncogene 31: 4577-4587, 2012.

13. Tsai MC, Spitale RC and Chang HY: Long intergenic noncoding RNAs: New links in cancer progression. Cancer Res 71: 3-7, 2011.

14. Bian EB, Ma CC, He XJ, Wang C, Zong G, Wang HL and Zhao B: Epigenetic modification of miR-141 regulates SKA2 by an endogenous 'sponge' HOTAIR in glioma. Oncotarget 7: 30610-30625, 2016.

15. Wang P, Liu YH, Yao YL, Li Z, Li ZQ, Ma J and Xue YX: Long non-coding RNA CASC2 suppresses malignancy in human gliomas by miR-21. Cell Signal 27: 275-282, 2015.

16. Wang Y, Wang Y, Li J, Zhang Y, Yin H and Han B: CRNDE, a long-noncoding RNA, promotes glioma cell growth and invasion through mTOR signaling. Cancer Lett 367: 122-128, 2015.

17. Zhao X, Wang P, Liu J, Zheng J, Liu Y, Chen J and Xue Y: Gas5 exerts tumor-suppressive functions in human glioma cells by targeting miR-222. Mol Ther 23: 1899-1911, 2015.

18. Li J, Bian EB, He XJ, Ma CC, Zong G, Wang HL and Zhao B: Epigenetic repression of long non-coding RNA MEG3 mediated by DNMT1 represses the 553 pathway in gliomas. Int $\mathrm{J}$ Oncol 48: 723-733, 2016.

19. Lin A, Li C, Xing Z, Hu Q, Liang K, Han L, Wang C, Hawke DH, Wang S, Zhang Y, et al: The LINK-A lncRNA activates normoxic HIF1 $\alpha$ signalling in triple-negative breast cancer. Nat Cell Biol 18: 213-224, 2016.

20. Le A, Cooper CR, Gouw AM, Dinavahi R, Maitra A, Deck LM, Royer RE, Vander Jagt DL, Semenza GL and Dang CV: Inhibition of lactate dehydrogenase A induces oxidative stress and inhibits tumor progression. Proc Natl Acad Sci USA 107: 2037-2042, 2010.

21. Esteller M: Non-coding RNAs in human disease. Nat Rev Genet 12: 861-874, 2011.

22. Mercer TR, Dinger ME and Mattick JS: Long non-coding RNAs: Insights into functions. Nat Rev Genet 10: 155-159, 2009.

23. Wilusz JE, Sunwoo H and Spector DL: Long noncoding RNAs: Functional surprises from the RNA world. Genes Dev 23: 1494-1504, 2009.

24. Cheetham SW, Gruhl F, Mattick JS and Dinger ME: Long noncoding RNAs and the genetics of cancer. Br J Cancer 108: 2419-2425, 2013.

25. Kung JT, Colognori D and Lee JT: Long noncoding RNAs: Past, present, and future. Genetics 193: 651-669, 2013.

26. Mattick JS: The genetic signatures of noncoding RNAs. PLoS Genet 5: e1000459, 2009.

27. Mattick JS and Makunin IV: Non-coding RNA. Hum Mol Genet 15: R17-R29, 2006.

28. Gupta RA, Shah N, Wang KC, Kim J, Horlings HM, Wong DJ, Tsai MC, Hung T, Argani P, Rinn JL, et al: Long non-coding RNA HOTAIR reprograms chromatin state to promote cancer metastasis. Nature 464: 1071-1076, 2010.

29. Yang F, Bi J, Xue X, Zheng L, Zhi K, Hua J and Fang G: Up-regulated long non-coding RNA H19 contributes to proliferation of gastric cancer cells. FEBS J 279: 3159-3165, 2012.

30. Zhang K, Sun X, Zhou X, Han L, Chen L, Shi Z, Zhang A, Ye M, Wang Q, Liu C, et al: Long non-coding RNA HOTAIR promotes glioblastoma cell cycle progression in an EZH2 dependent manner. Oncotarget 6: 537-546, 2015.

31. Han Y, Wu Z, Wu T, Huang Y, Cheng Z, Li X, Sun T, Xie X, Zhou Y and Du Z: Tumor-suppressive function of long noncoding RNA MALAT1 in glioma cells by downregulation of MMP2 and inactivation of ERK/MAPK signaling. Cell Death Dis 7: e2123, 2016. 
32. Shi M, Cui J, Du J, Wei D, Jia Z, Zhang J, Zhu Z, Gao Y and Xie K: A novel KLF4/LDHA signaling pathway regulates aerobic glycolysis in and progression of pancreatic cancer. Clin Cancer Res 20: 4370-4380, 2014.

33. Yang Y, Su D, Zhao L, Zhang D, Xu J, Wan J, Fan S and Chen M: Different effects of LDH-A inhibition by oxamate in non-small cell lung cancer cells. Oncotarget 5: 11886-11896, 2014.

34. Zhao D, Zou SW, Liu Y, Zhou X, Mo Y, Wang P, Xu YH, Dong B, Xiong Y, Lei QY, et al: Lysine-5 acetylation negatively regulates lactate dehydrogenase $\mathrm{A}$ and is decreased in pancreatic cancer. Cancer Cell 23: 464-476, 2013.

35. Chesnelong C, Chaumeil MM, Blough MD, Al-Najjar M, Stechishin OD, Chan JA, Pieper RO, Ronen SM, Weiss S, Luchman HA, et al: Lactate dehydrogenase A silencing in IDH mutant gliomas. Neuro-oncol 16: 686-695, 2014.

36. Cai Z, Zhao JS, Li JJ, Peng DN, Wang XY, Chen TL, Qiu YP, Chen PP, Li WJ, Xu LY, et al: A combined proteomics and metabolomics profiling of gastric cardia cancer reveals characteristic dysregulations in glucose metabolism. Mol Cell Proteomics 9: $2617-2628,2010$
37. Crane CA, Austgen K, Haberthur K, Hofmann C, Moyes KW, Avanesyan L, Fong L, Campbell MJ, Cooper S, Oakes SA, et al: Immune evasion mediated by tumor-derived lactate dehydrogenase induction of NKG2D ligands on myeloid cells in glioblastoma patients. Proc Natl Acad Sci USA 111: 12823-12828, 2014.

38. Kim J, Han J, Jang Y, Kim SJ, Lee MJ, Ryu MJ, Kweon GR and Heo JY: High-capacity glycolytic and mitochondrial oxidative metabolisms mediate the growth ability of glioblastoma. Int J Oncol 47: 1009-1016, 2015.

39. Fantin VR, St-Pierre J and Leder P: Attenuation of LDH-A expression uncovers a link between glycolysis, mitochondrial physiology, and tumor maintenance. Cancer Cell 9: 425-434, 2006. 